\title{
State of Charge Estimation for Lithium-Ion Battery by Using Dual Square Root Cubature Kalman Filter
}

\author{
Luping Chen, Liangjun Xu, and Ruoyu Wang \\ School of Automation, Beijing University of Posts and Telecommunications, Beijing 100876, China \\ Correspondence should be addressed to Luping Chen; luping_chen@bupt.edu.cn
}

Received 30 June 2017; Revised 22 October 2017; Accepted 6 December 2017; Published 24 December 2017

Academic Editor: Marek Lefik

Copyright (C) 2017 Luping Chen et al. This is an open access article distributed under the Creative Commons Attribution License, which permits unrestricted use, distribution, and reproduction in any medium, provided the original work is properly cited.

\begin{abstract}
The state of charge (SOC) plays an important role in battery management systems (BMS). However, SOC cannot be measured directly and an accurate state estimation is difficult to obtain due to the nonlinear battery characteristics. In this paper, a method of SOC estimation with parameter updating by using the dual square root cubature Kalman filter (DSRCKF) is proposed. The proposed method has been validated experimentally and the results are compared with dual extended Kalman filter (DEKF) and dual square root unscented Kalman filter (DSRUKF) methods. Experimental results have shown that the proposed method has the most balance performance among them in terms of the SOC estimation accuracy, execution time, and convergence rate.
\end{abstract}

\section{Introduction}

In recent years, energy conservation and emission reduction have become a significant issue in uninterruptible power system (UPS) industry. Due to the superior energy density, long cycle life, and low pollution, lithium-ion batteries instead of lead-acid batteries have become popular as energy storage devices. Despite its overall advantages, lithium-ion batteries require battery management systems (BMS) to choose the control strategy of discharging or charging current in the electrical circuit to safeguard the battery performance. The state of charge (SOC) estimation is one of the most key techniques in the BMS, since it is essential to enhance the utilization efficiency of battery energy, lengthen the battery lifetime, and prevent the permanent battery damage [1]. However, SOC cannot be measured directly and it is estimated from measurable quantities such as the battery current and voltage.

Several methods of estimating the battery SOC have been proposed including ampere-hour counting (Ah), open circuit voltage (OCV), artificial neural networks (ANN), fuzzy logic $(\mathrm{FL})$, particle filter $(\mathrm{PF})$, and improved Kalman filters [2-15]. Among them, Ah method is one of the most commonly used methods. It can be simply implemented by integrating the battery current over time [2]. However, the reliability of SOC estimation cannot be maintained when errors accumulate over the operation time or the initial value of SOC is unknown. OCV method is another commonly used method. However, it needs the battery to be rested for a long period to estimate the battery's OCV [3]. ANN and FL methods can estimate SOC with an arbitrary initial SOC value $[4,5]$. However, the SOC estimation accuracy strongly relies on the quantity and quality of the training data set. A limited training data set may give a poor result and reduce the applicability of this method. In order to improve the accuracy of SOC estimation, the model-based estimation methods have been further developed. The extended Kalman filter (EKF) method transforms the nonlinear system of charging and discharging into a linear system by linearizing the nonlinear function on the basis of the first-order Taylor series expansion [6]. However, not only the instability of the filter and the lack of robustness due to the linearization process but also the errorprone calculation of the Jacobian matrices can be listed as the shortcomings of the EKF method. In order to make up for the shortcomings of EKF, the unscented Kalman filter (UKF) and cubature Kalman filter (CKF) methods are proposed, based on the unscented transform [7] and radial-spherical cubature rule [8], respectively. They need not to linearize the nonlinear process and calculate the Jacobian matrix. Thus, the two methods have a higher accuracy over the EKF in SOC estimation. The PF method is a Monte-Carlo-based approximation method that uses a set of weighted random 
samples to approximate the system states [9]. Although PF method can improve accuracy effectively, its computational complexity is enormous [10]. Thus, PF method is not suitable for on-line SOC estimation. The accuracy of the model-based methods depends on not only the establishment of the battery equivalent model but also the battery model parameters. In the process of charging and discharging, the battery model parameters are not constant, which will lead to the drifting of the SOC estimation result [11]. In order to solve the problem, some researchers consider two Kalman filters structure to estimate SOC [12-15]. The dual EKF (DEKF) method is a combination of two EKFs, in which the SOC is estimated by the first EKF, and the parameter is estimated by the second EKF [12-14]. Compared with the single filter structure, the SOC accuracy of two filters is improved greatly. The dual square root UKF method is also proposed to estimate the SOC and the battery parameters [15]. However, there is no comparison about SOC estimation accuracy, execution time, and convergence rate between the DEKF and DSRUKF methods.

In this paper, in order to estimate the battery SOC with parameters updating and give a balance method among the SOC estimation accuracy, execution time, and convergence rate, the dual square root cubature Kalman filter (DSRCKF) method is proposed, which has not been mentioned in the existing literature. The square root aspect of the filter improves the numerical stability by ensuring that the state covariance is always semipositive definite [16]. CKF only requires $2 n$ cubature points while UKF needs $2 n+1$ sigma points [17]. As a result, CKF seems to have a better computational speed than UKF theoretically. Therefore, proposed method fusion frame in CKF. The SOC of battery is estimated by the first square root cubature Kalman filter, and the battery parameters are updated by the second square root cubature Kalman filter. The proposed DSRCKF method has been verified experimentally compared with the widely used method of DEKF and the recent research method of DSRUKF. It has shown that proposed method owns the most balanced SOC estimation result among them according to trade-offs between accuracy, execution time, and convergence rate.

The outline of this paper is as follows. Section 2 presents the popular model of lithium-ion battery and the method of parameter updating. Section 3 presents the proposed DSRCKF method for SOC estimation and parameters updating in detail. Section 4 presents the experimental setup and the discussions of the proposed method. Section 5 concludes this paper.

\section{Battery Modeling and Parameter Updating}

2.1. Battery Modeling. The second-order RC equivalent circuit model is used as the battery model in this paper. This model is simple and effective which can be applied easily in engineering [18]. Figure 1 shows the equivalent circuit model which consists of a voltage source, a series resistor, and two sets of parallel resistor-capacitor circuits. $U_{\text {oc }}$ represents the battery OCV, $R_{0}$ represents the battery internal resistance, $R_{1}$ and $C_{1}$ are the charge-transfer resistor and electric double-layer capacitor, respectively, which

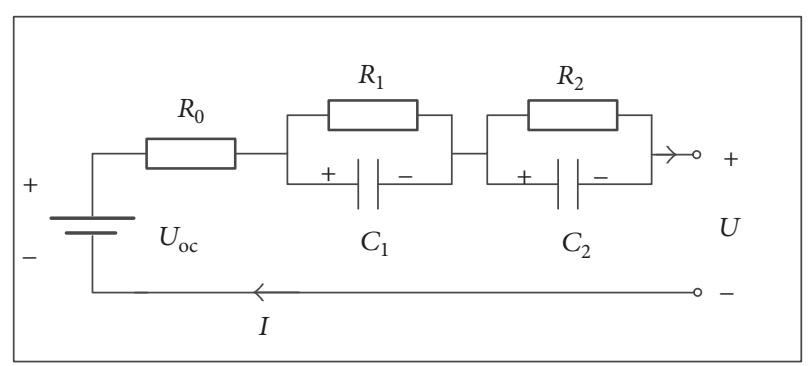

Figure 1: The second-order RC equivalent circuit model.

represent the charge-transfer reaction, and $R_{2}$ and $C_{2}$ are the diffusion resistor and diffusion capacitor, respectively, which represent the diffusion phenomenon caused by the grade of the concentration of the electrolyte near the electrode.

According to the battery circuit equations and SOC model definition, the discrete state space equation of battery model can be expressed as follows [19]:

$$
\begin{aligned}
{\left[\begin{array}{c}
\mathrm{SOC}_{k+1} \\
U_{1, k+1} \\
U_{2, k+1}
\end{array}\right]=} & {\left[\begin{array}{ccc}
1 & 0 & 0 \\
0 & 1-\frac{T_{s}}{R_{1} C_{1}} & 0 \\
0 & 0 & 1-\frac{T_{s}}{R_{2} C_{2}}
\end{array}\right] \cdot\left[\begin{array}{c}
\mathrm{SOC}_{k} \\
U_{1, k} \\
U_{2, k}
\end{array}\right] } \\
& +\left[\begin{array}{c}
-\frac{\eta T_{s}}{C_{A}} \\
\frac{T_{s}}{C_{1}} \\
\frac{T_{s}}{C_{2}}
\end{array}\right] \cdot i_{k}+w_{k} \\
U_{k}= & U_{\mathrm{OC}}\left(\mathrm{SOC}_{k}\right)-U_{1, k}-U_{2, k}-i_{k} \cdot R_{0}+v_{k},
\end{aligned}
$$

where $w_{k}$ and $v_{k}$ represent the process noise and measuring noise of the battery system, respectively. $U_{\mathrm{OC}}\left(\mathrm{SOC}_{k}\right)$ can be obtained by experiment of the relation between OCV and SOC.

2.2. Parameter Updating. According to Kirchhoff voltage law, the electrical behavior of the equivalent circuit in the frequency domain can be expressed as follows:

$$
\begin{aligned}
G(s) & =\frac{\Delta U(s)}{I(s)}=\frac{U_{\mathrm{oc}}-U}{I(s)} \\
& =R_{0}+\frac{R_{1}}{1+R_{1} C_{1} s}+\frac{R_{2}}{1+R_{2} C_{2} s} .
\end{aligned}
$$

By using the bilinear transformation as shown in (3) for the transfer function $G(s)$, the discrete transfer function of the Battery system with sampling time $T$ can be expressed as (4).

$$
\mathrm{s}=\frac{2}{T} \cdot \frac{1-z^{-1}}{1+z^{-1}}
$$


where $z$ is the discretization operation.

$$
\begin{aligned}
G\left(z^{-1}\right)= & R_{0}+\frac{R_{1} T\left(1+z^{-1}\right)}{\left(T+2 R_{1} C_{1}\right)+\left(T-2 R_{1} C_{1}\right) z^{-1}} \\
& +\frac{R_{2} T\left(1+z^{-1}\right)}{\left(T+2 R_{2} C_{2}\right)+\left(T-2 R_{2} C_{2}\right) z^{-1}} \\
= & \frac{b_{0}+b_{1} z^{-1}+b_{2} z^{-2}}{1+a_{1} z^{-1}+a_{2} z^{-2}} .
\end{aligned}
$$

According to (4), the relation between the battery parameters $\left(R_{0}, R_{1}, R_{2}, C_{1}, C_{2}\right)$ and the indirect parameters $\left(a_{1}, a_{2}, b_{0}, b_{1}, b_{2}\right)$ is as follows:

$$
\begin{aligned}
& R_{0}=\frac{b_{0}-b_{1}+b_{2}}{1-a_{1}+a_{2}} \\
& R_{1} C_{1} R_{2} C_{2}=\frac{1-a_{1}+a_{2}}{4\left(1+a_{1}+a_{2}\right)} T^{2} \\
& R_{1} C_{1}+R_{2} C_{2}=\frac{1-a_{2}}{1+a_{1}+a_{2}} T \\
& R_{2}\left(R_{1} C_{1}\right)+R_{1}\left(R_{2} C_{2}\right)+R_{0}\left(R_{1} C_{1}+R_{2} C_{2}\right) \\
& \quad=\frac{b_{0}-b_{2}}{1+a_{1}+a_{2}} T \\
& R_{0}+R_{1}+R_{2}=\frac{b_{0}+b_{1}+b_{2}}{1+a_{1}+a_{2}} .
\end{aligned}
$$

Equation (4) can also be transformed to the following differential equation:

$$
\begin{aligned}
\Delta U(k)= & -a_{1} \Delta U(k-1)-a_{2} \Delta U(k-2)+b_{0} I(k) \\
& +b_{1} I(k-1)+b_{2} I(k-2),
\end{aligned}
$$

where $I(k)$ and $\Delta U(k)$ indicate the system input and output, respectively.

For lithium-ion batteries, the battery parameters change very slowly [11]. So we model them as constants with some small perturbations. Due to the fact that the state space equation of battery parameters cannot be directly established for filtering, it needs to solve the indirect parameters in order to obtain the battery parameters. The state space equation of indirect parameters estimation can be expressed as follows:

$$
\begin{aligned}
\theta(k+1) & =\theta(k)+r_{k} \\
\Delta U(k) & =C_{k} \theta_{k}+e_{k}
\end{aligned}
$$

where $\theta=\left[-a_{1},-a_{2}, b_{0}, b_{1}, b_{2}\right]^{T}, C_{k}=\left[\Delta U_{k-1}, \Delta U_{k-2}, I_{k}, I_{k-1}\right.$, $\left.I_{k-2}\right]$, and $r_{k}$ and $e_{k}$ represent the small perturbations and measuring noise, respectively.

\section{SOC Estimation by Using DSRCKF Method}

The DSRCKF framework contains two square root cubature Kalman filters (SRCKFs). The first SRCKF is a state filter used for estimating the SOC, the second SRCKF is a weight filter used for updating the battery parameters $\left(R_{0}, R_{1}, R_{2}, C_{1}, C_{2}\right)$. Due to the difficulty in establishing the state space equation of battery parameters directly, this paper uses the weight filter to estimate the indirect parameters $\left(a_{1}, a_{2}, b_{0}, b_{1}, b_{2}\right)$ by the state space equation (7), then the updating battery parameters are solved by transform equation (5). Finally, this paper uses the state filter to estimate the SOC with battery parameter updating by state space equation (1).

The whole structure of SOC estimation has been shown in Figure 2. Firstly, the relation between OCV and SOC should be established for the state space equation (1). And initialize the SOC value and battery parameters for the state filter. At the same time, the initial battery parameter should be transformed to initial indirect parameters used for the weight filter by equation (5). After the initialization, two filters interact in each iteration loop. In each iteration step, the state filter uses the last iteration value of the weight filter, and the weight filter also uses the last iteration value of the state filter. By following this procedure, the estimation accuracy can be maintained even if the battery parameters have shifted.

The DSRCKF detailed algorithm is described from (8) to (37). The state space equation of the SOC and indirect parameters are presented by (8) and (9), respectively. Equation (10) represents the relation between battery parameters and indirect parameters. The detailed DSRCKF method by using the cubature rule [8] is presented as follows.

$$
\begin{aligned}
x(k+1) & =f(x(k), u(k), \varepsilon(k))+w_{k} \\
y(k) & =h(x(k), u(k), \varepsilon(k))+v_{k} \\
\theta(k+1) & =\theta(k)+r_{k} \\
d(k) & =g(x(k), u(k), \theta(k))+e_{k} \\
\varepsilon(k) & =\varphi(\theta(k)),
\end{aligned}
$$

where $w_{k}, v_{k}, r_{k}, e_{k}$ are independent, zero-mean, and Gaussian noises with covariance matrices $P_{w}, P_{v}, P_{r}, P_{e}$, respectively.

(1) Time Updated for State

(1) Factorize the following:

$$
P_{x, k-1}^{+}=S_{x, k-1}^{+}\left(S_{x, k-1}^{+}\right)^{T} .
$$

(2) Obtain the cubature points $(i=1,2, \ldots, 2 N)$ :

$$
\xi_{x, i, k-1}^{+}=S_{x, k-1}^{+} \xi_{x, i}+\hat{x}_{k-1}^{+} .
$$

(3) Obtain the propagated cubature points:

$$
\gamma_{x, i, k}^{-}=f\left(\xi_{x, i, k-1}^{+}, \widehat{\varepsilon}_{k-1}^{+}\right) .
$$

(4) Estimate the predicted state:

$$
\widehat{x}_{k}^{-}=\frac{1}{2 n} \sum_{i=1}^{2 n} \gamma_{x, i, k}^{-}
$$

where $n$ is the dimension of the state vector. 


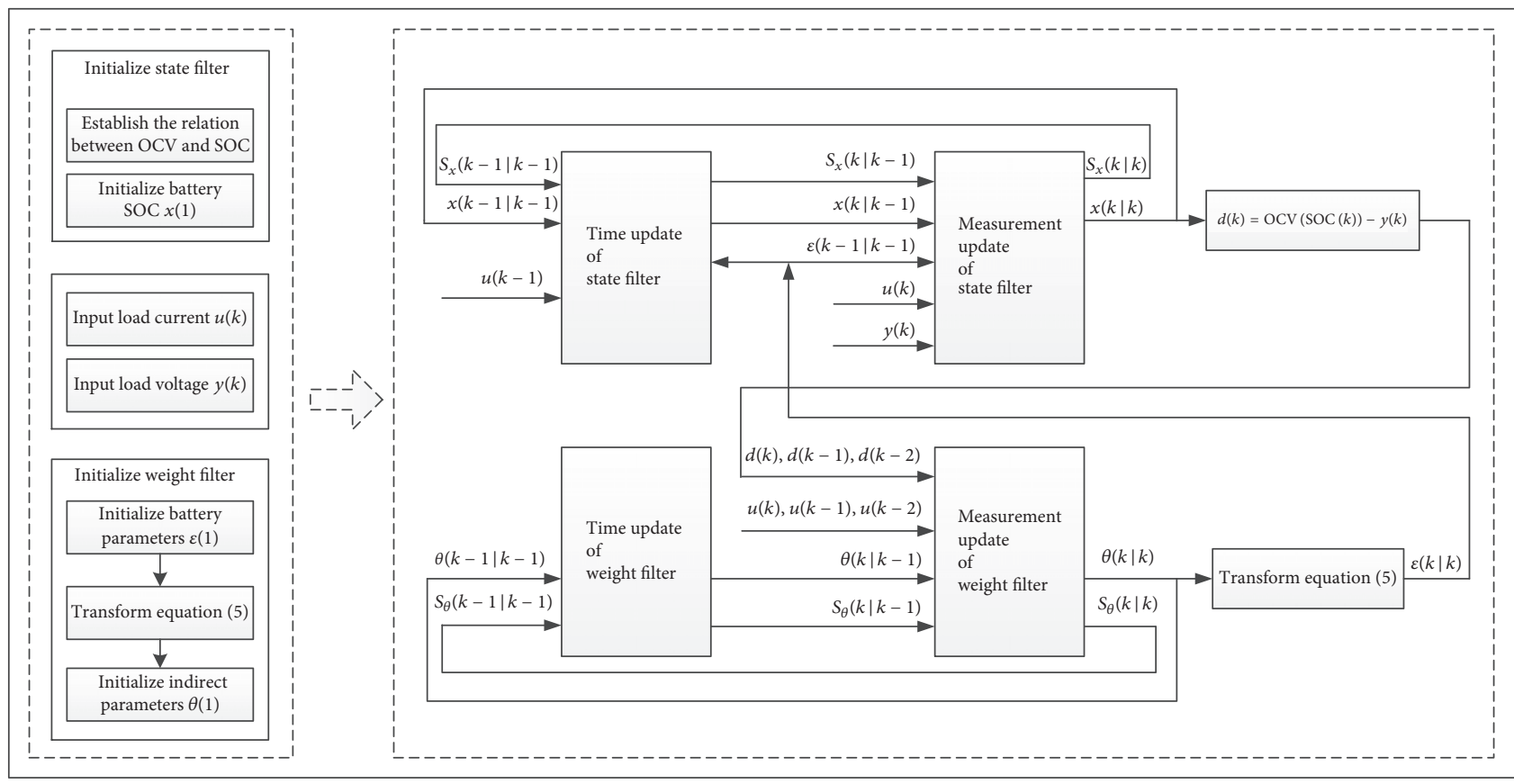

FIGURE 2: The framework of SOC estimation by using proposed DSRCKF method.

(5) Square root factor of the predicted error covariance is as follows:

$\varphi_{x, k}=\frac{1}{\sqrt{2 n}}\left[\begin{array}{llll}\gamma_{x, 1, k}^{-}-\widehat{x}_{k}^{-} & \gamma_{x, 2, k}^{-}-\widehat{x}_{k}^{-} & \cdots & \gamma_{x, 2 n, k}^{-}-\widehat{x}_{k}^{-}\end{array}\right]$

$S_{x, k}^{-}=\operatorname{Tria}\left(\left[\begin{array}{ll}\varphi_{x, k} & \sqrt{Q_{x, k-1}}\end{array}\right]\right)$,

where $Q_{x, k-1}$ is the state system noise at time $k-1$. Tria() is the function to compute the square root factor of the matrix.

\section{(2) Time Updated for Weight}

(1) Factorize the following:

$$
P_{\theta, k-1}^{+}=S_{\theta, k-1}^{+}\left(S_{\theta, k-1}^{+}\right)^{T}
$$

(2) Obtain the cubature points $(i=1,2, \ldots, 2 M)$ :

$$
\xi_{\theta, i, k-1}^{+}=S_{\theta, k-1}^{+} \xi_{\theta, i}+\widehat{\theta}_{k-1}^{+}
$$

(3) Obtain the propagated cubature points:

$$
\gamma_{\theta, i, k}^{-}=\xi_{\theta, i, k-1}^{+} \text {. }
$$

(4) Estimate the predicted weight:

$$
\widehat{\theta}_{k}^{-}=\frac{1}{2 m} \sum_{i=1}^{2 m} \gamma_{\theta, i, k}^{-},
$$

where $m$ is the dimension of the indirect parameter vector.
(5) Square root factor of the predicted error covariance is as follows:

$\varphi_{\theta, k}$

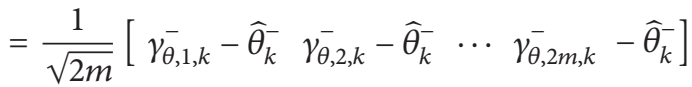

$S_{\theta, k}^{-}=\operatorname{Tria}\left(\left[\begin{array}{ll}\varphi_{\theta, k} & \sqrt{Q_{\theta, k-1}}\end{array}\right]\right)$,

where $Q_{\theta, k-1}$ is the weight system noise at time $k-1$.

(3) Measurement Updated for State

(1) Obtain the cubature points $(i=1,2, \ldots, 2 N)$ :

$$
\xi_{x, i, k}^{-}=S_{x, k}^{-} \xi_{i}+\widehat{x}_{k}^{-}
$$

(2) Obtain the propagated cubature points:

$$
\chi_{x, i, k}^{-}=h\left(\xi_{x, i, k}^{-}, \widehat{\varepsilon}_{k-1}^{+}\right) \text {. }
$$

(3) Estimate the predicted measurement:

$$
\widehat{z}_{x, k}^{-}=\frac{1}{2 n} \sum_{i=1}^{2 n} \chi_{x, i, k}^{-} .
$$

(4) Square root factor of the innovation covariance is as follows:

$$
\begin{aligned}
& \eta_{x, k}^{-}=\frac{1}{\sqrt{2 n}} \\
& \cdot\left[\begin{array}{llll}
\chi_{x, 1, k}^{-}-\widehat{z}_{x, k}^{-} & \chi_{x, 2, k}^{-}-\widehat{z}_{x, k}^{-} & \cdots & \chi_{x, 2 n, k}^{-}-\widehat{z}_{x, k}^{-}
\end{array}\right], \\
& S_{x, z z, k}^{-}=\operatorname{Tria}\left(\eta_{x, k}^{-} \sqrt{R_{x, k}}\right) .
\end{aligned}
$$


(5) The cross-covariance matrix is as follows:

$$
\begin{aligned}
& \chi_{x, k}^{-} \\
& =\frac{1}{\sqrt{2 n}}\left[\begin{array}{llll}
\xi_{x, 1, k}^{-}-\widehat{x}_{k}^{-} & \xi_{x, 2, k}^{-}-\widehat{x}_{k}^{-} & \cdots & \xi_{x, 2 n, k}^{-}-\widehat{x}_{k}^{-}
\end{array}\right], \\
& P_{x, x z, k}^{-}=\chi_{x, k}^{-}\left(\eta_{x, k}^{-}\right)^{T} \text {. }
\end{aligned}
$$

(6) The estimated Kalman gain is as follows:

$$
W_{x, k}=\frac{\left(P_{x, x z, k}^{-} /\left(S_{x, z z, k}^{-}\right)^{T}\right)}{S_{x, z z, k}^{-}} .
$$

(7) The estimated state vector is as follows:

$$
\hat{x}_{k}^{+}=\widehat{x}_{k}^{-}+W_{x, k}\left(z_{x, k}^{+}-\widehat{z}_{x, k}^{-}\right) .
$$

(8) Square root factor of the related error covariance is as follows:

$$
S_{x, k}^{+}=\operatorname{Tria}\left(\left[\chi_{x, k}^{-}-W_{x, k} \eta_{x, k}^{-} \quad W_{x, k} \sqrt{R_{x, k}}\right]\right) .
$$

\section{(4) Measurement Update for Weight}

(1) Obtain the cubature points $(i=1,2, \ldots, 2 M)$ :

$$
\xi_{\theta, i, k}^{-}=S_{\theta, k}^{-} \xi_{i}+\widehat{\theta}_{k}^{-} .
$$

(2) Obtain the propagated cubature points:

$$
\chi_{\theta, i, k}^{-}=g\left(\xi_{\theta, i, k}^{-}\right) .
$$

(3) Estimate the predicted measurement:

$$
\widehat{z}_{\theta, k}^{-}=\frac{1}{2 m} \sum_{i=1}^{2 m} \bar{\chi}_{\theta, i, k}^{-} .
$$

(4) Square root factor of the innovation covariance is as follows:

$$
\begin{aligned}
\eta_{\theta, k}^{-} & =\frac{1}{\sqrt{2 m}} \\
\cdot & {\left[\begin{array}{llll}
\chi_{\theta, 1, k}^{-}-\widehat{z}_{\theta, k}^{-} & \chi_{\theta, 2, k}^{-}-\widehat{z}_{\theta, k}^{-} & \cdots & \chi_{\theta, 2 m, k}^{-}-\widehat{z}_{\theta, k}^{-}
\end{array}\right], } \\
S_{\theta, z z, k}^{-} & =\operatorname{Tria}\left(\begin{array}{lll}
\eta_{\theta, k}^{-} & \left.\sqrt{R_{\theta, k}}\right) .
\end{array}\right.
\end{aligned}
$$

(5) The cross-covariance matrix is as follows:

$\chi_{\theta, k}^{-}$

$$
\begin{aligned}
& \quad=\frac{1}{\sqrt{2 m}}\left[\begin{array}{lllll}
\xi_{\theta, 1, k}^{-}-\widehat{\theta}_{k}^{-} & \xi_{\theta, 2, k}^{-}-\widehat{\theta}_{k}^{-} & \cdots & \xi_{\theta, 2 m, k}^{-}-\widehat{\theta}_{k}^{-}
\end{array}\right], \\
& P_{\theta, x z, k}^{-}=\chi_{\theta, k}^{-}\left(\eta_{\theta, k}^{-}\right)^{T} .
\end{aligned}
$$

TABLE 1: Initial battery parameters.

\begin{tabular}{lc}
\hline Parameters & Values \\
\hline$R_{0}$ & $0.1766 \Omega$ \\
$R_{1}$ & $0.0186 \Omega$ \\
$C_{1}$ & $1432 \mathrm{~F}$ \\
$R_{2}$ & $0.0222 \Omega$ \\
$C_{2}$ & $62303 \mathrm{~F}$ \\
\hline
\end{tabular}

(6) The estimated Kalman gain is as follows:

$$
W_{\theta, k}=\frac{\left(P_{\theta, x z, k}^{-} /\left(S_{\theta, z z, k}^{-}\right)^{T}\right)}{S_{\theta, z z, k}^{-}} .
$$

(7) The estimated weight vector is as follows:

$$
\widehat{\theta}_{k}^{+}=\widehat{\theta}_{k}^{-}+W_{\theta, k}\left(z_{\theta, k}^{+}-\widehat{z}_{\theta, k}^{-}\right) .
$$

(8) Square root factor of the related error covariance is as follows:

$$
S_{\theta, k}^{+}=\operatorname{Tria}\left(\left[\overline{\chi_{\theta, k}^{-}}-W_{\theta, k} \eta_{\theta, k}^{-} W_{\theta, k} \sqrt{R_{\theta, k}}\right]\right) .
$$

(5) Transformation between Battery Parameter Vector and Indirect Parameter Vector

$$
\widehat{\varepsilon}_{k}^{+}=\varphi\left(\widehat{\theta}_{k}^{+}\right) .
$$

\section{Experiments and Analysis}

4.1. Experimental Setup. To validate the proposed method, a battery test bench has been set up in Figure 3. It consists of tested battery (18650 lithium-ion battery, 4.2 V/2.5 Ah), a DC power supply for battery charging (IV-3605, IVYTECH, China), a DC electronic load for battery discharging (LK-15A, LUKANG Electronics, China), a control board for voltage and current data acquisition, relays for switch of charging and discharging, and a host computer for monitoring and storing discharging voltage and current data.

4.2. Pretest for SOC Estimation. In order to estimate battery SOC, the initial battery parameters $\left(R_{0}, R_{1}, R_{2}, C_{1}, C_{2}\right)$ and OCV-SOC equation need to be determined, respectively. Reasonable initial parameter values will help the filter converge faster. As shown in Table 1, the initial battery parameters are extracted from a sequence of pulse discharging experiments [20].

As shown in Figure 4, the relation between OCV and SOC has been constructed by using pulse discharging experimental data [20]. Considering the nonlinear relation between OCV and SOC, this paper uses the fifth-order polynomial to fit the relation curve [21]. It is clear that the relation between $\mathrm{OCV}$ and SOC can be expressed well by the following:

$$
\begin{aligned}
U_{\mathrm{oc}}(\mathrm{SOC})= & 16.51 * \mathrm{SOC}^{5}-46.48 * \mathrm{SOC}^{4}+48.22 \\
& * \mathrm{SOC}^{3}-22.08 * \mathrm{SOC}^{2}+5.077 \\
& * \mathrm{SOC}+2.962 .
\end{aligned}
$$




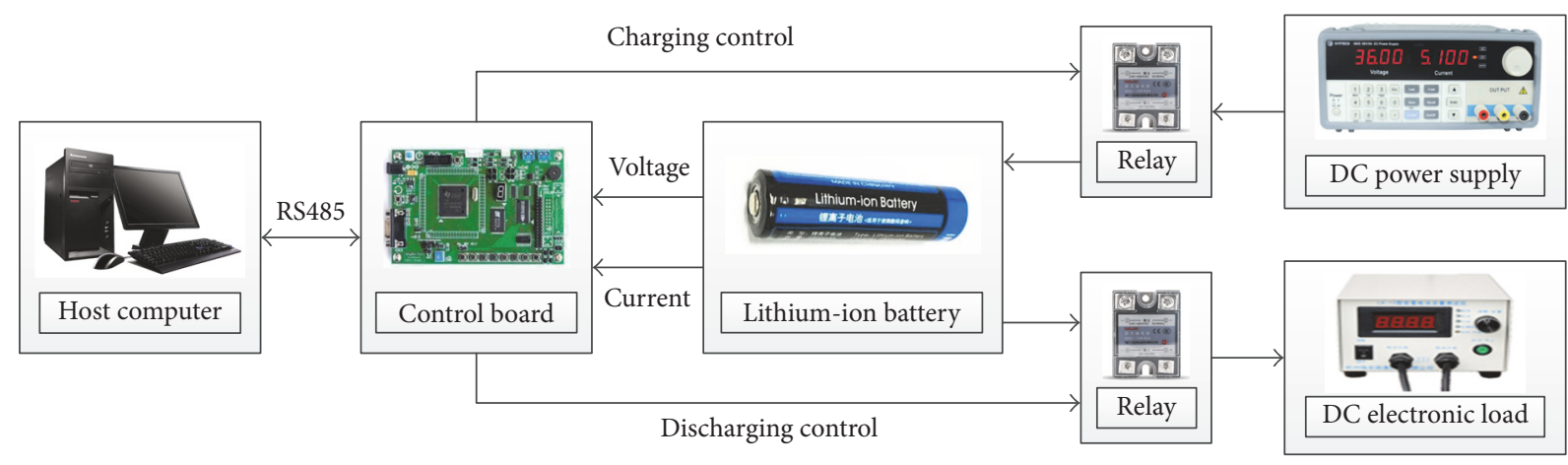

FIGURE 3: Configuration of the battery test bench.

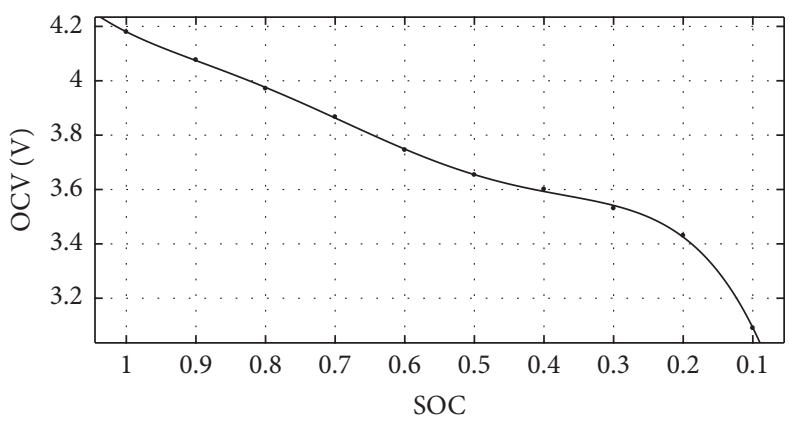

Figure 4: The relation between OCV and SOC.

4.3. Result and Discussion. In this section, the battery is discharging with constant load current $(0.5 \mathrm{~A})$ after the battery has been fully charged up to $100 \%$ SOC. The battery discharging is terminated when the battery terminal voltage reaches the cut-off voltage. In the experiment, a set of voltage data with 10 seconds sampling period were collected for analysis and the discharging curve has a nonlinear characteristic as shown in Figure 5(a). Ampere-hour counting is considered to calculate the reference value of the SOC estimation for the performance comparison in Figure 5(b).

The proposed method is compared with the widely used DEKF and the standard DSRUKF methods in terms of estimation accuracy, computational complexity, and convergence rate. To estimate the SOC accuracy, an initial SOC value of 1 and the initial battery parameters in Table 1 were used for the DEKF, DSRUKF, and DSRCKF methods, approximately equal to the real measure value. All three methods by using DEKF (green line), DSRUKF (blue line), and DSRCKF (red line) can be used to estimate the SOC value well in Figure 6(a), but, in Figure 6(b), there is a difference in accuracy compared with the reference SOC. As shown in Figure 6(c), the SOC estimation values are changed rapidly in the initial stage (from 0 to $50 \mathrm{~s}$ ) of the estimation process due to the drifting of the actual initial battery parameters. But the SOC error has a trend to get smaller and more stable in the middle stage due to the tracking characteristics of Kalman filter. The SOC estimation values are fluctuated in the final stage (from $12000 \mathrm{~s}$ to $16000 \mathrm{~s}$ ) due to the nonlinearity of the battery discharging which can be referred to in Figure 5(a). As shown in Figure 6(d), the accuracies of SOC estimation of DSRUKF and DSRCKF are similar and the difference between them can be ignored. It can be found that the DSRUKF and DSRCKF methods can reduce the SOC estimation error in comparison with the DEKF method. In addition, the proposed DSRCKF method can reduce the fluctuation of SOC estimation compared with the DEKF method. The execution time shows the computational complexity of the applied nonlinear state estimation methods in the Matlab environment. Thereby, the execution time is calculated as the time taken by the individual methods to estimate the battery states during one full discharge scenario. The SOC comparison results, including root mean square error (RMSE), maximum absolute error, and execution time, are summarized in Table 2.

Different initial SOC errors in terms of $10 \%$ SOC intervals from 0 to 1 are considered for the proposed DSRCKF method. The convergence criteria are $2 \%$ referenced the SOC error fluctuation when the initial SOC error is 1 in Figure 6(b). For example, the experimental results are shown in Figure 7(a) when the initial SOC are reset to 0.8 (red dotted line), 0.6 (blue dotted line), and 0.4 (green dotted line), respectively. The convergence rates are all fast in the early stage and then slow down gradually with the convergence process, but the convergence criteria are met in the end in Figure 7(b).

To further compare the convergence performance between the DEKF (green line), DSRUKF (blue line), and the proposed DSRCKF (red line) method, the initial SOC value is reset to 0.6 in Figure 8, significantly different from the experimental SOC values. In Figure 8(b), the convergence rate of the DSRUKF and DSRCKF are slower than that of 


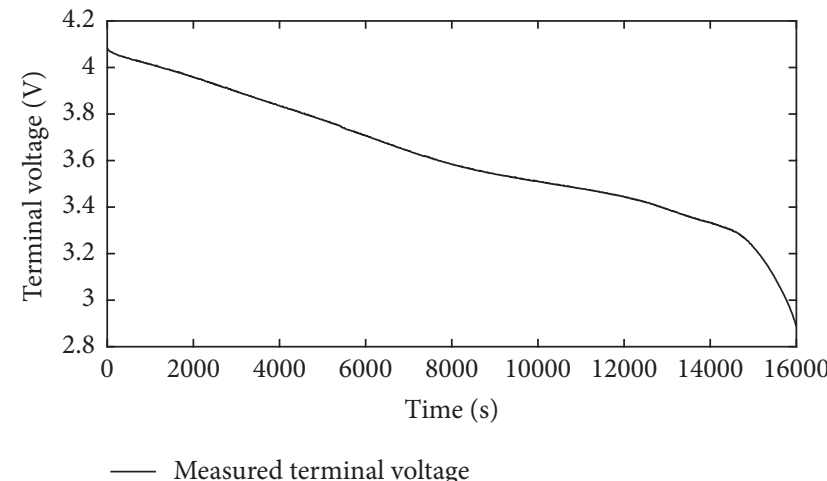

(a) Battery terminal voltage response

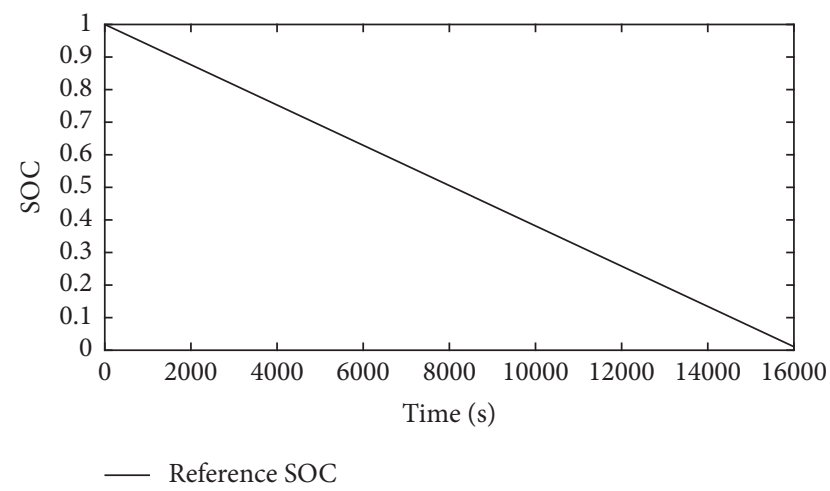

(b) Reference SOC by Ah method

FIGURE 5: The measured voltage and reference SOC.

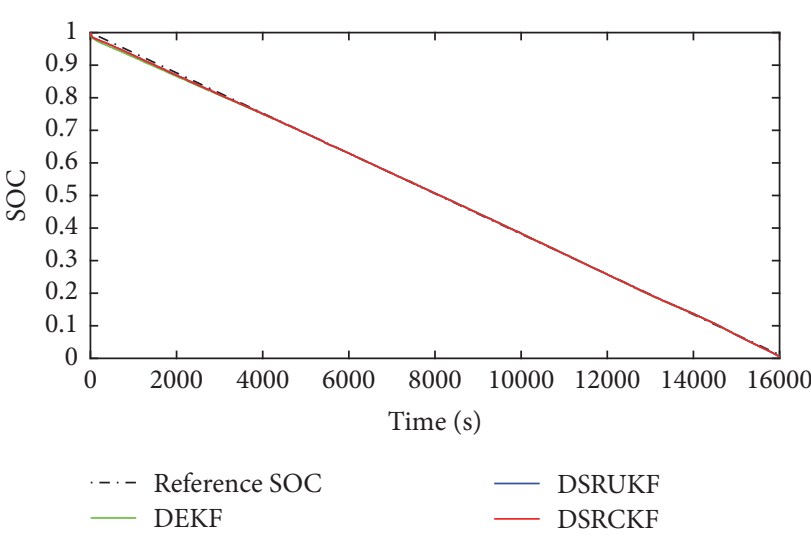

(a) SOC estimation

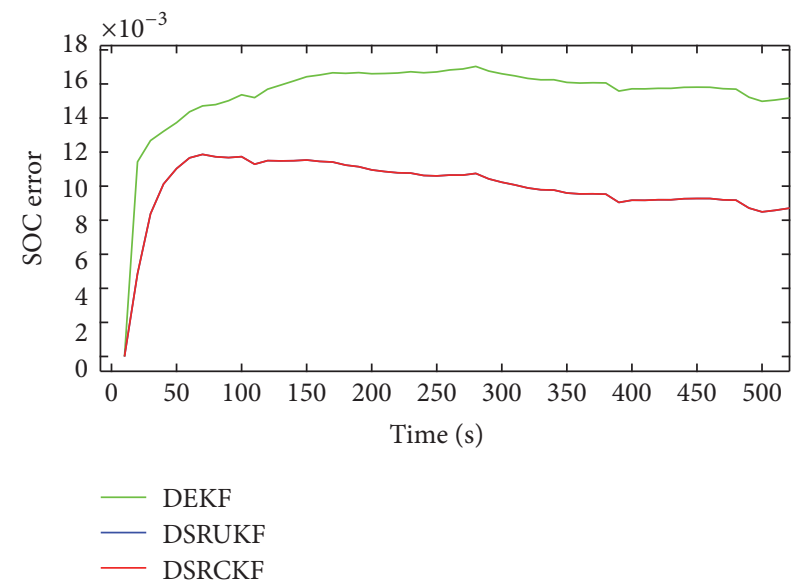

(c) Zoom figure for (b)

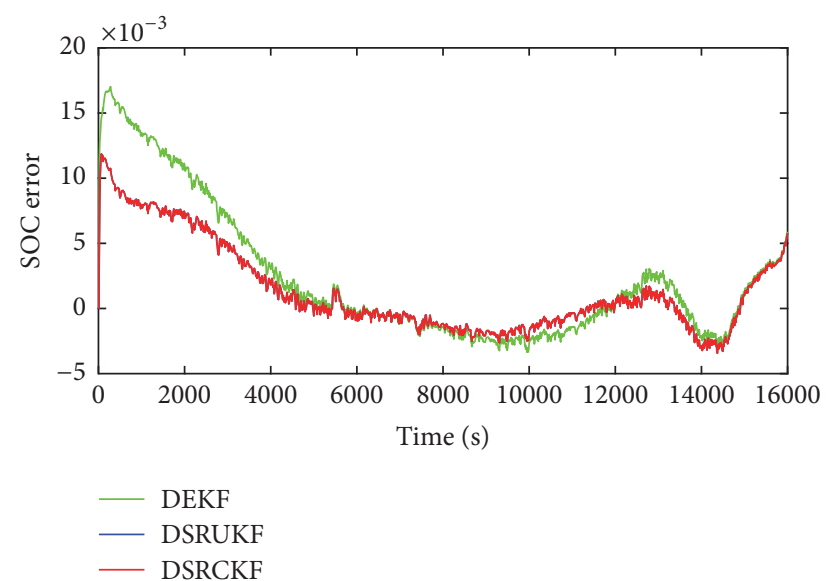

(b) SOC estimation error

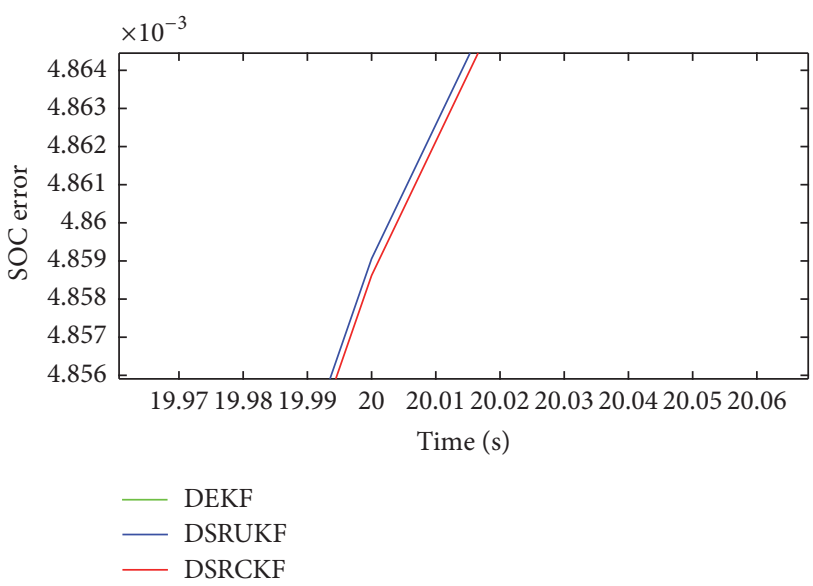

(d) Zoom figure for (c)

FIGURE 6: SOC estimation by DEKF (green line), DSRUKF (blue line), and DSRCKF (red line).

TABLE 2: Comparison of SOC estimation and execution time.

\begin{tabular}{lccc}
\hline Method & RMSE & Maximum absolute error & Execution time (s) \\
\hline DEKF & 0.0057 & 0.0170 & 0.3995 \\
DSRUKF & 0.0037 & 0.0119 & 1.1379 \\
DSRCKF & 0.0037 & 0.0119 & 0.8883 \\
\hline
\end{tabular}




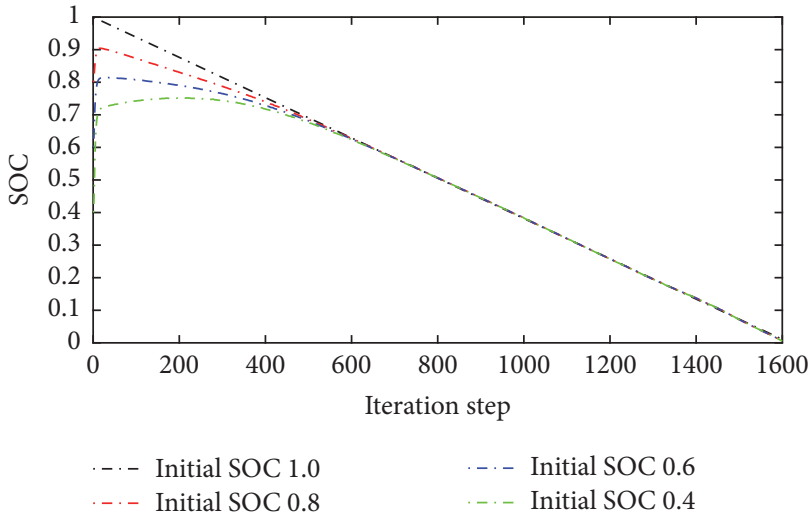

(a) SOC estimation when the initial SOC is $0.8,0.6$, and 0.4 , respectively

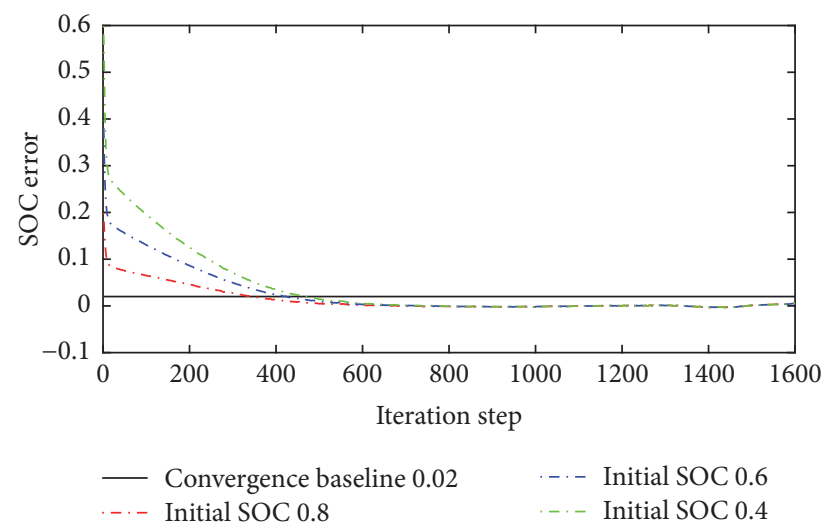

(b) SOC error when the initial SOC is $0.8,0.6$, and 0.4 , respectively

FIGURE 7: Convergence analysis by using the proposed DSRCKF method.

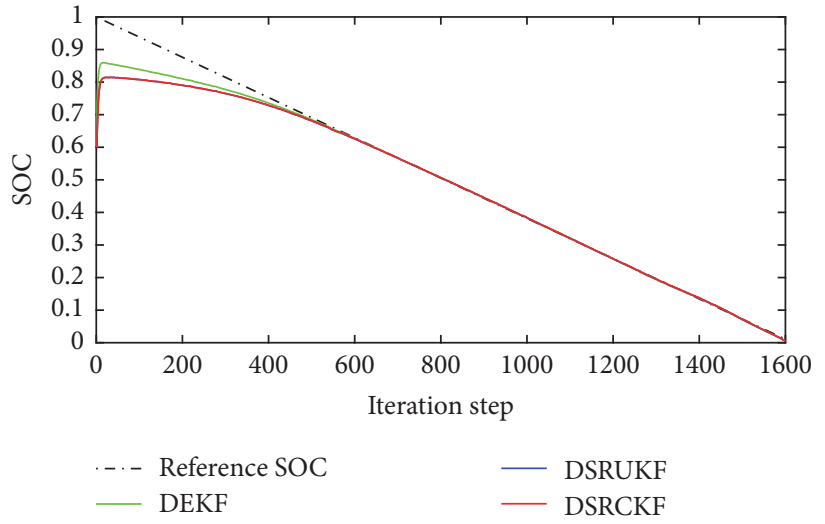

(a) SOC estimation when the initial SOC is 0.6

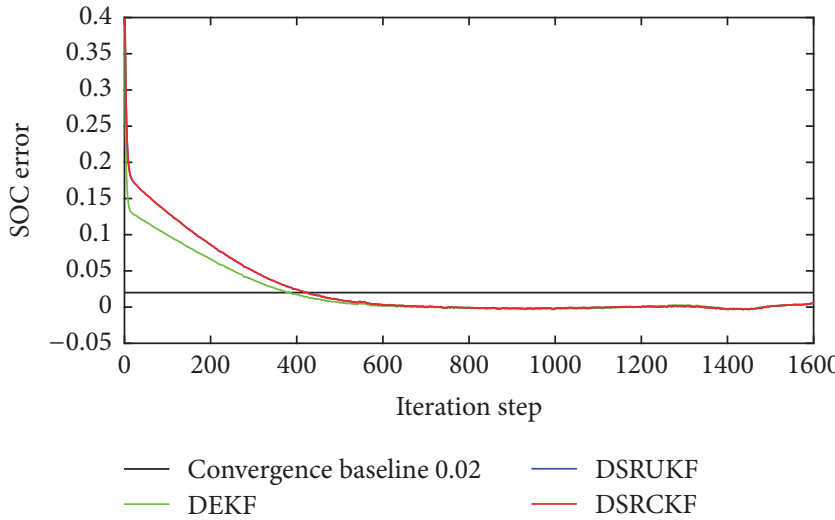

(b) SOC error when the initial SOC is 0.6

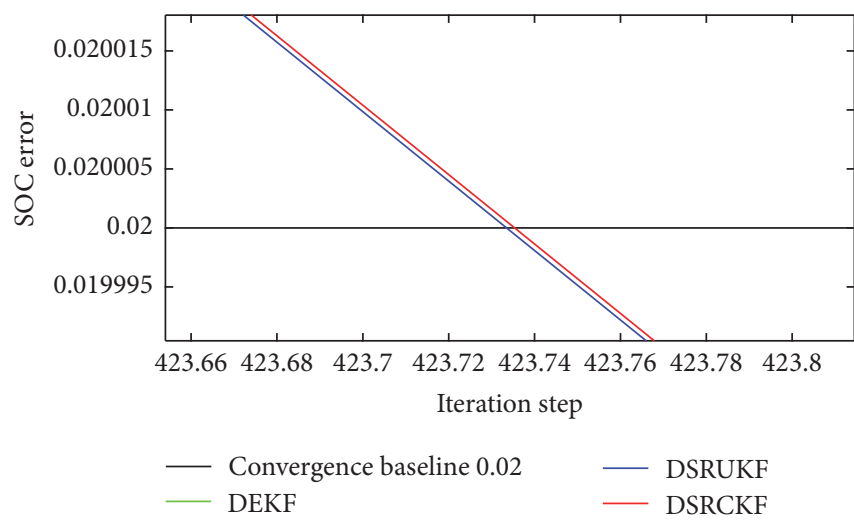

(c) Zoom figure for (b)

FIGURE 8: Comparison of convergence between the DEKF, DSRUKF, and DSRCKF methods.

the DEKF method. In Figure 8(c), The DSRUKF method has a slight advantage in convergence rate compared with the DSRCKF method, but the advantage can be ignored due to the microscopic distinction.

A complete convergence analysis comparison between DEKF, DSRUKF, and DSRCKF is summarized in Table 3. It shows that all three methods have good convergent performance. The convergence rates of the DSRCKF and
DSRUKF methods are approximate but slower than that of DEKF method.

A complete analysis of advantages between DEKF, DSRUKF, and DSRCKF method for SOC estimation is summarized in Table 4. A, B, and C mean the excellent classes which from high to low, respectively. The DSRUKF and the proposed DSRCKF methods have a higher SOC estimation accuracy than the DEKF method. The DSRCKF method has 
TABLE 3: Comparison of convergence analysis.

\begin{tabular}{lccc}
\hline Initial SOC & \multicolumn{3}{c}{$\begin{array}{c}\text { Convergence iteration (step) } \\
\text { DSRUKF }\end{array}$} \\
\hline $100 \%$ & 1 & 1 & 1 \\
$90 \%$ & 195 & 233 & 233 \\
$80 \%$ & 300 & 347 & 347 \\
$70 \%$ & 351 & 396 & 396 \\
$60 \%$ & 381 & 423 & 423 \\
$50 \%$ & 404 & 444 & 444 \\
$40 \%$ & 424 & 464 & 464 \\
$30 \%$ & 441 & 491 & 491 \\
$20 \%$ & 455 & 508 & 508 \\
$10 \%$ & 480 & 518 & 518 \\
$0 \%$ & 495 & 529 & 529 \\
\hline
\end{tabular}

TABLE 4: Comparison of the overall performance analysis.

\begin{tabular}{lccc}
\hline & DEKF & DSRUKF & DSRCKF \\
\hline SOC accuracy & $\mathrm{C}$ & $\mathrm{A}$ & $\mathrm{A}$ \\
Execution time & $\mathrm{A}$ & $\mathrm{C}$ & $\mathrm{B}$ \\
Convergence rate & $\mathrm{A}$ & $\mathrm{B}$ & $\mathrm{B}$ \\
\hline
\end{tabular}

lower execution time than that of DSRUKF, but higher than that of DEKF. The convergence rates of the DSRCKF and DSRUKF methods are approximate, but a little slower than that of DEKF method.

\section{Conclusion}

In this paper, a novel SOC estimation method with parameters updating by using the DSRCKF has been proposed. The second-order RC equivalent circuit model is applied to simulate the nonlinear behaviors of lithium-ion battery. The state space equation of SOC estimation is utilized for the first SRCKF. The state space equation of parameters updating is utilized for the second SRCKF. In each iteration loop process, the two filters interact with each other. The experimental results demonstrate that the DSRCKF method has a better overall performance than the DSRUKF method with the lower execution time, the approximate SOC estimation accuracy, and convergence rate. Despite the disadvantages of the execution time and convergence rate, the DSRCKF method has a higher SOC estimation accuracy than the DEKF method. To sum up, if the accuracy of the SOC estimation is the priority, the proposed DSRCKF method would be the most balanced estimation method among them according the trade-offs among accuracy, execution time, and convergence rate.

In the future research, the influence of dynamic load current will be further discussed. As an application, the proposed method will also be considered under different operating environments.

\section{Conflicts of Interest}

The authors declare that there are no conflicts of interest regarding the publication of this paper.

\section{References}

[1] H. Chaoui, "State of Charge and State of Health Estimation for Lithium Batteries Using Recurrent Neural Networks," IEEE Transactions on Vehicular Technology, vol. 66, no. 10, pp. 87738783, 2017.

[2] F. Codeca, S. M. Savaresi, and G. Rizzoni, "On battery State of Charge estimation: A new mixed algorithm," in Proceedings of the 17th IEEE International Conference on Control Applications, CCA, pp. 102-107, USA, September 2008.

[3] S.-J. Huang, B.-G. Huang, and F.-S. Pai, "An approach to measurements of electrical characteristics of lithium-ion battery with open-circuit voltage function," IET Power Electronics, vol. 5, no. 9, pp. 1968-1975, 2012.

[4] C. Cai, D. Du, Z. Liu, and J. Ge, "State-of-charge (SOC) estimation of high power Ni-MH rechargeable battery with artificial neural network," in Proceedings of the 9th International Conference on Neural Information Processing, ICONIP 2002, pp. 824-828, Singapore, November 2002.

[5] S. Maila, Embedded-system controlled fuzzy logic expert system to estimate battery-SOC [M.sc. thesis], Lamar University, 2008.

[6] Y. Fang, X. Cheng, and Y. Yin, "SOC estimation of lithium-ion battery packs based on thevenin model," Applied Mechanics and Materials, vol. 299, pp. 211-215, 2013.

[7] J. Zhou and X. Zhang, "Power battery SOC estimation with combination method based on UKF and open circuit voltage," International Journal of Electric and Hybrid Vehicles, vol. 6, no. 3, pp. 215-226, 2014.

[8] Z. Cheng, J.-K. Lv, J.-G. Liu, and L. Wang, "Application of equivalent hysteresis model in estimaion of state of charge of lithium-ion battery," Hunan Daxue Xuebao/Journal of Hunan University Natural Sciences, vol. 42, no. 4, pp. 63-70, 2015.

[9] J. Du, Y. Wang, and C. Wen, "Li-ion battery SOC estimation using particle filter based on an equivalent circuit model," in Proceedings of the 2013 10th IEEE International Conference on Control and Automation, ICCA 2013, pp. 580-585, June 2013.

[10] W. Wang, D. Wang, X. Wang et al., "Comparison of Kalman Filter-based state of charge estimation strategies for Li-Ion batteries," in Proceedings of the 2016 IEEE Transportation Electrification Conference and Expo, ITEC 2016, USA, June 2016.

[11] J. L. Huang, Y. N. Wang, Z. F. Wang, F. L. Han, and L. G. Li, "The experiments of dual Kalman filter in lithium battery SOC estimation," Applied Mechanics Materials, pp. 1509-1512, 2013.

[12] S. Lee, J. Kim, and B. H. Cho, "Maximum pulse current estimation for high accuracy power capability prediction of a Li-Ion battery," Microelectronics Reliability, vol. 55, no. 3-4, pp. 572-581, 2015.

[13] V. H. Duong, N. T. Tran, W. Choi, and D.-W. Kim, "State estimation technique for VRLA batteries for automotive applications," Journal of Power Electronics, vol. 16, no. 1, pp. 238-248, 2016.

[14] N. Tran, A. Khan, and W. Choi, "State of Charge and State of Health Estimation of AGM VRLA Batteries by Employing a Dual Extended Kalman Filter and an ARX Model for Online Parameter Estimation," Energies, vol. 10, no. 1, p. 137, 2017.

[15] H. Aung and K. S. Low, "Temperature dependent state-ofcharge estimation of lithium ion battery using dual spherical 
unscented Kalman filter," IET Power Electronics, vol. 8, no. 10, pp. 2026-2033, 2015.

[16] X. Zhang, Y. Wang, C. Liu, and Z. Chen, "A novel approach of remaining discharge energy prediction for large format lithiumion battery pack," Journal of Power Sources, vol. 343, pp. 216-225, 2017.

[17] I. Arasaratnam and S. Haykin, "Cubature Kalman filters," Institute of Electrical and Electronics Engineers Transactions on Automatic Control, vol. 54, no. 6, pp. 1254-1269, 2009.

[18] X. Zhang, J. Wu, and G. Kang, "SOC estimation of Lithium battery by UKF algorithm based on dynamic parameter model," in Proceedings of the 13th International Conference on Ubiquitous Robots and Ambient Intelligence, URAI 2016, pp. 945-950, China, August 2016.

[19] Z. Cheng, Q.-Y. Zhang, and Y.-H. Zhang, "Online state-ofcharge estimation of LI-ion battery based on the second-order RC model," Advanced Materials Research, vol. 805-806, pp. 1659-1663, 2013.

[20] Y.-T. Luo, B. Xie, and X.-C. He, "Parameter identification and SOC estimation of lithium-ion battery pack used in electric vehicles," Huanan Ligong Daxue Xuebao/Journal of South China University of Technology (Natural Science), vol. 40, no. 12, pp. 79-85, 2012.

[21] B. Xia, Z. Sun, R. Zhang, and Z. Lao, "A cubature particle filter algorithm to estimate the state of the charge of lithium-ion batteries based on a second-order equivalent circuit model," Energies, vol. 10, no. 4, p. 457, 2017. 


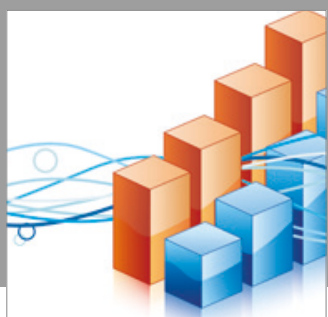

Advances in

Operations Research

vatersals

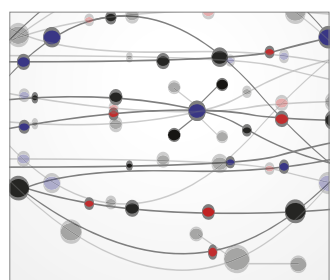

\section{The Scientific} World Journal
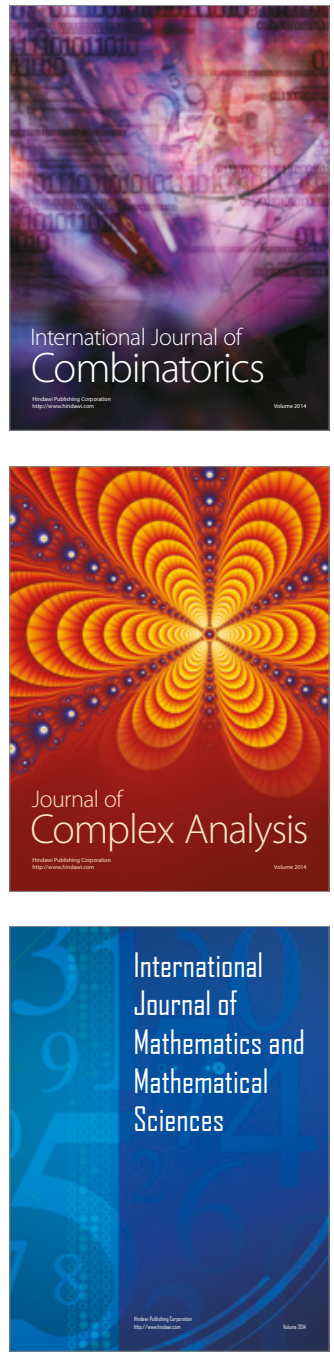
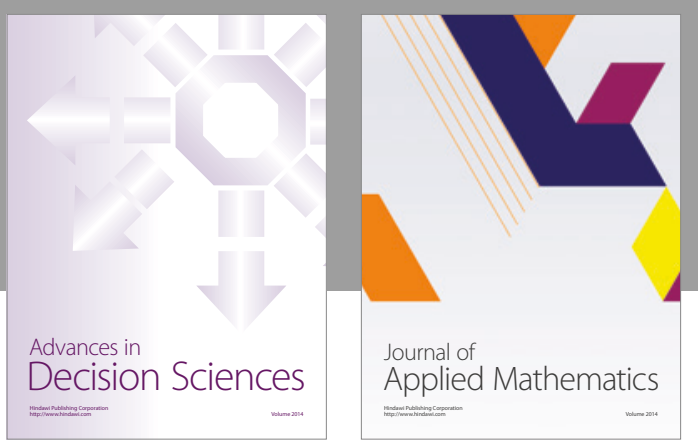

Algebra

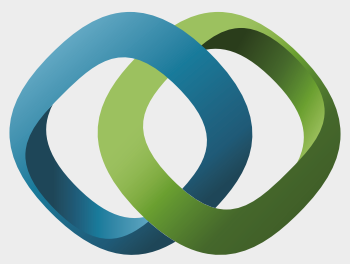

\section{Hindawi}

Submit your manuscripts at

https://www.hindawi.com
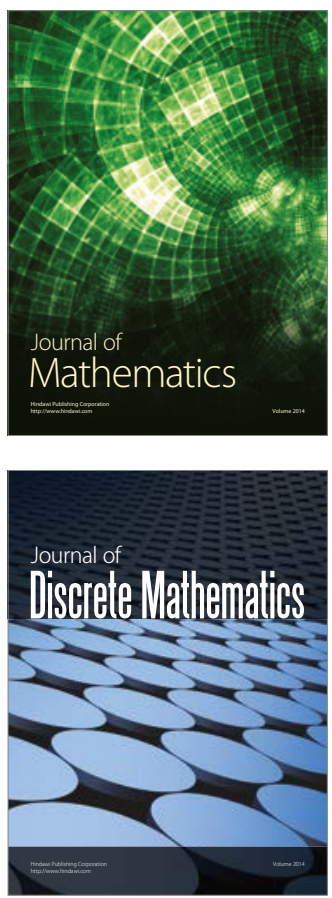

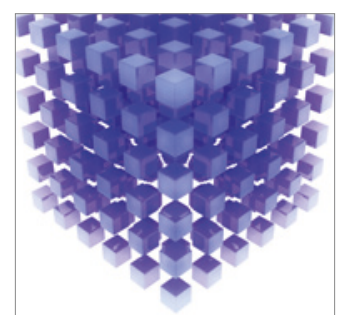

Mathematical Problems in Engineering
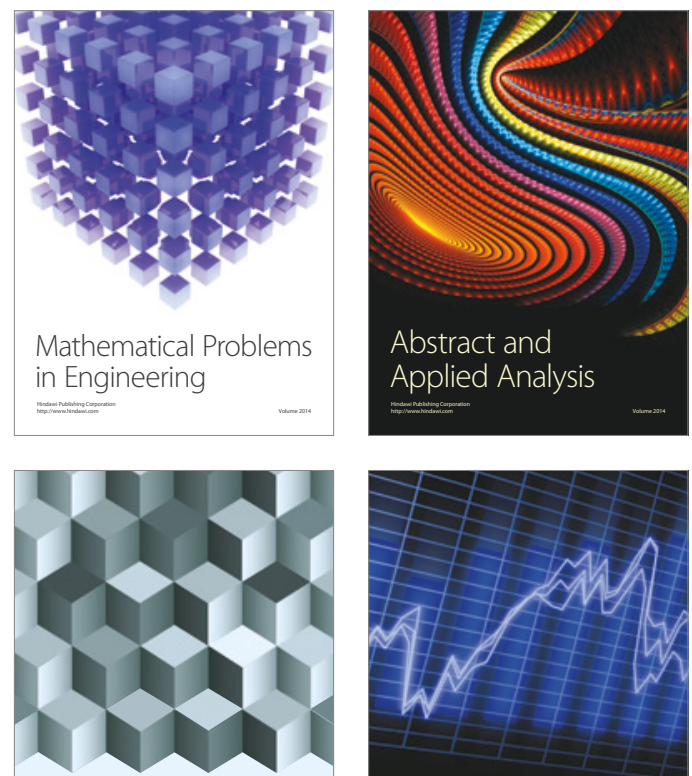

Journal of

Function Spaces

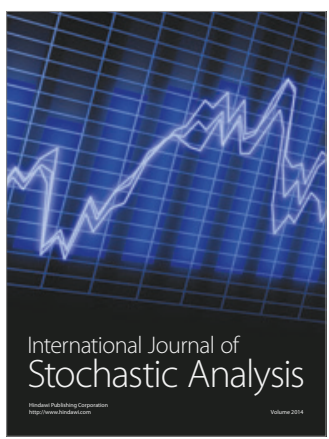

Probability and Statistics
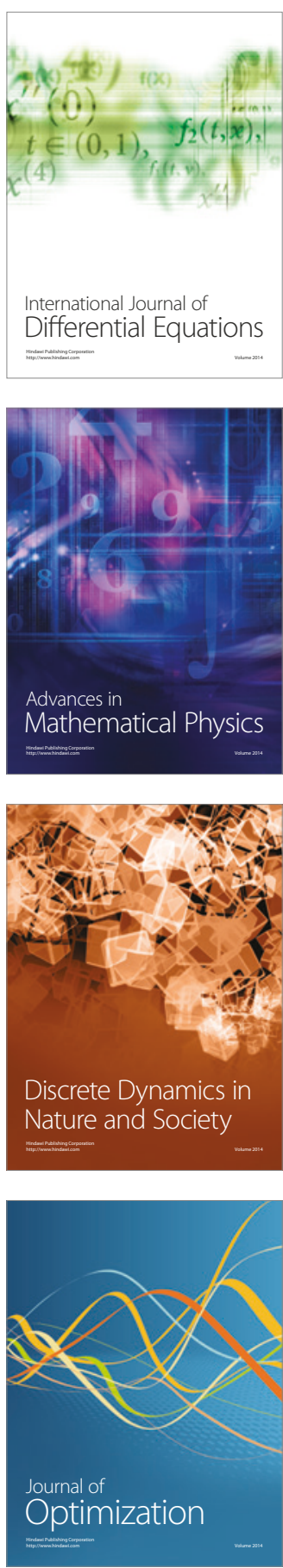